\title{
Evidence for Genetic Linkage Between a Polymorphism in the Adenosine 2A Receptor and Panic Disorder
}

\author{
Steven P Hamilton 1,6,7, Susan L Slager², Ada Baisre de Leon', Gary A Heiman', Donald F Klein', \\ Susan E Hodge ${ }^{1,4}$, Myrna M Weissman ',3, Abby J Fyer' and James A Knowles*, ',5 \\ 'Department of Psychiatry, College of Physicians and Surgeons at Columbia University and the New York State Psychiatric Institute, USA; \\ ${ }^{2}$ Department of Health Sciences Research, Mayo Clinic, USA; ${ }^{3}$ Division of Epidemiology, Mailman School of Public Health, Columbia University, \\ USA; ${ }^{4}$ Division of Biostatistics, USA; ${ }^{5}$ Columbia Genome Center, College of Physicians and Surgeons at Columbia University, USA; ${ }^{6}$ Department of \\ Psychiatry, University of California, San Francisco, USA
}

\begin{abstract}
Data from clinical and behavioral pharmacological studies have implicated adenosine in anxiety behaviors, while genetic studies have suggested that adenosine receptors may be associated with panic disorder. We have undertaken an analysis of several DNA sequence variations in the adenosine 2A receptor (ADORA2A) in a large sample of panic disorder pedigrees. Individuals from 70 panic disorder pedigrees, and 83 child-parent 'trios', were genotyped at five single-nucleotide polymorphisms (SNPs) in and near the ADORA2A gene and were analyzed for genetic linkage and association. Linkage analysis revealed elevated LOD scores for a silent substitution (I083C/T, SNP-4) in the second coding exon. This SNP has been previously reported to be associated with panic disorder. We observed a maximal heterogeneity LOD score of $2.98(\theta=0)$ under a recessive genetic model and narrow diagnostic model. Other SNPs showed no evidence for linkage. Association tests were not significant for any of the five ADORA2A SNPs. When SNP haplotypes were assessed in the triads with TRANSMIT, one 3-marker haplotype (SNPs I, 4, 5) was nominally significantly associated with panic disorder ( $p=0.029$ ). Pairwise estimations of linkage disequilibrium between the SNPs showed strong patterns of linkage disequilibrium across the ADORA2A locus. Analyses carried out by broadening the panic disorder phenotype to include agoraphobia continued to support linkage to ADORA2A. Our findings provide evidence for a susceptibility locus for panic disorder, and possibly including agoraphobia, either within the ADORA2A gene or in a nearby region of chromosome 22, and serves as the first successful candidate gene replication study in panic disorder.

Neuropsychopharmacology (2004) 29, 558-565, advance online publication, 10 December 2003; doi: I 0. I038/sj.npp. I 3003 I I
\end{abstract}

Keywords: ADORA2A; adenosine receptor; panic disorder; genetic; association; linkage; SNP

\section{INTRODUCTION}

Panic disorder (PD) is a common psychiatric condition defined by recurrent panic attacks and anticipatory anxiety, with a lifetime prevalence of $1-3 \%$. A 2.6- to 20 -fold relative risk to the first-degree relatives of probands with $\mathrm{PD}$ suggests a familial component to this disorder (Knowles and Weissman, 1995), while twin studies show that about $40 \%$ of the liability towards PD consists of heritable factors (Hettema et al, 2001). The etiology of PD is currently unknown.

*Correspondence: Dr JA Knowles, New York State Psychiatric Institute, Unit \#28, 1051 Riverside Drive, New York City, NY 10032, USA, Tel: + I 212543 5867, Fax: + I 212543621 I,

E-mail: jak8@columbia.edu

Current address: Department of Psychiatry, University of California, San Francisco.

Received 3I January 2003; revised 0 I July 2003; accepted 07 August 2003

Online publication: 19 August 2003 at http://www.acnp.org/citations/ Npp081903030048/default.pdf
Adenosine is a purine nucleoside that plays multiple physiological roles, with the most prominent being in the cardiovascular and central nervous systems (Guieu et al, 1998). Adenosine has been long known to be a neuromodulator, with both inhibitory and excitatory functions (Moreau and Huber, 1999). Although not stored or released as a classical neurotransmitter, adenosine is involved in the fine-tuning of other neuromodulators (Sebastiao and Ribeiro, 2000). All four of the known adenosine receptors are expressed in the brain, particularly the expression of the $2 \mathrm{~A}$ adenosine receptor. Animal work has implicated this receptor in a number of behaviors, including locomotion, anxiety, aggression, reward, nociception, sleep, seizures, psychotic-like behaviors, and aging. In the absence of a selective $2 \mathrm{~A}$ receptor ligand, there has been limited research into the role of these receptors in human psychopathology. These observations suggest a role for adenosine in Parkinson's disease, schizophrenia, anxiety disorders, and Alzheimer's disease (Moreau and Huber, 1999).

The receptors for adenosine are potentially attractive candidate genes for PD. Caffeine has long been recognized 
as one of the agents that can precipitate panic attacks (Charney et al, 1985). Caffeine is an antagonist of the four adenosine receptors and is most potent for the type $2 \mathrm{~A}$ adenosine receptor (ADORA2A). The ADORA2A locus is located on human chromosome 22q11.23, while the A1, $\mathrm{A} 2 \mathrm{~B}$, and $\mathrm{A} 3$ receptors are located on $1 \mathrm{q} 32.1,17 \mathrm{p} 12$, and $1 \mathrm{p} 13.2$, respectively. Male mice in which this gene is disrupted show increased anxiety-like behaviors, and also aggressive behavior (Ledent et al, 1997). Previous work has shown that a silent coding polymorphism $(1083 \mathrm{C} / \mathrm{T}$, or SNP-4 in this study) in exon 2 in ADORA2A is associated with PD (Deckert et al, 1998). A Japanese group tried to replicate this finding in 87 PD patients, but found no association (Yamada et al, 2001). Given the possible importance of adenosine receptor function in panic, we proposed to study the role of single-nucleotide polymorphisms (SNPs) in or near the ADORA2A gene in a population of patients with PD.

For the study reported here, we utilized five SNPs in the ADORA2A region in a family-based design. Our goal is to ascertain any linkage or association between $\mathrm{PD}$ and ADORA2A. We do this by testing for linkage and association with each SNP and with multilocus haplotypes.

\section{METHODS AND MATERIALS}

\section{Subjects}

Families were recruited from several sources, including anxiety clinics, therapists specializing in treating anxiety disorders, and anxiety disorder support groups/associations. Initially, families with at least three affected persons were asked to participate. Participants underwent interviews utilizing the Schedule for Affective Disorders and Schizophrenia-Lifetime Version for Anxiety Disorders, Revised (Mannuzza et al, 1986) and the Family Informant Schedule and Criteria (Mannuzza et al, 1985), from which DSM-III-R diagnoses of PD with recurrent spontaneous panic attack \pm agoraphobia (APA) were derived, as previously described (Fyer and Weissman, 1999; Knowles et al,
1998). In this study, 70 multiplex families and 83 triads consisting of proband, mother, and father were genotyped. The protocol was approved by the institutional review board of the New York State Psychiatric Institute. All interviews of probands and relatives were performed after obtaining signed informed consent. Relatives were contacted with the permission of the proband. The majority of families are Caucasian, of Western European background, and currently reside in the United States.

\section{DNA Analysis}

Preparation of the DNA samples was as described elsewhere (Knowles et al, 1998). A total of 14 SNPs were chosen from public databases (http://www.ncbi.nlm.nih.gov/SNP/, http:// snp.cshl.org/) or the literature (Deckert et al, 1998). Nine were nonpolymorphic in our population. All liquid handling was performed using the TECAN Genesis robotic sample processing system with Gemini software. SNPs were detected using fluorescence polarization, a technique that distinguishes the polymorphic base of an SNP by the template-directed incorporation of a dye-labeled dideoxynucleotide onto an oligonucleotide primer that anneals just $5^{\prime}$ to the polymorphic base (Chen et al, 1999). This homogenous reaction was performed in three stages (Hamilton et al, 2001). Briefly, in the first step, PCR reactions of $5 \mu$ containing $200 \mathrm{nM}$ of the forward and reverse primers (Table 1), $20 \mathrm{ng}$ DNA template, $50 \mu \mathrm{M}$ dNTPs, $1 \mathrm{M}$ anhydrous betaine (Sigma, St Louis), $50 \mathrm{mM}$ $\mathrm{KCl}, 20 \mathrm{mM}$ Tris- $\mathrm{HCl}$ ( $\mathrm{pH} 8.3$ ), $2.5 \mathrm{mM} \mathrm{MgCl}_{2}$, and $0.25 \mathrm{U}$ Platinum Taq DNA polymerase (GibcoBRL, Rockville) were performed. Primers were designed using Primer3 software (Rozen and Skaletsky, 1998), and manufactured by LifeTech (Frederick, MD). Using a touchdown protocol, samples were cycled at $94^{\circ} \mathrm{C}$ for $3 \mathrm{~min}$, followed by seven cycles of $94^{\circ} \mathrm{C}$ for $30 \mathrm{~s}, 65-59^{\circ} \mathrm{C}$ for $30 \mathrm{~s}$ (decreased by $1^{\circ} \mathrm{C}$ intervals per cycle), and $72^{\circ} \mathrm{C}$ for $30 \mathrm{~s}$, followed by 38 cycles of $90^{\circ} \mathrm{C}$ for $30 \mathrm{~s}, 58^{\circ} \mathrm{C}$ for $30 \mathrm{~s}$, and $72^{\circ} \mathrm{C}$ for $30 \mathrm{~s}$, with a final $10 \mathrm{~min}$ at $72^{\circ} \mathrm{C}$ on an MJ Research PTC-225 Thermal Cycler in 384well plates (MJ Research, Waltham, MA). The excess

Table I Polymorphic Loci Near or Within The ADORA2A Gene, With Allele Frequencies and Heterozygosity

\begin{tabular}{|c|c|c|c|c|c|c|c|c|c|}
\hline SNP & SNP & AP000355 ${ }^{a}$ & PCR primers (F/R) & FP-TDI probe & $\begin{array}{c}\text { Amplicon } \\
\text { (bp) }\end{array}$ & Effect & $d b S N P^{b}$ & $\begin{array}{l}\text { All. freq. } \\
(\%)\end{array}$ & Heterozyg. \\
\hline SNP-I & $\mathrm{G} / \mathrm{T}$ & 47922 & $\begin{array}{l}\text { ctccttggcttctcaccatc } \\
\text { ctccattggttgttgaagca }\end{array}$ & caccctgacttggaggatcttt & 135 & $5^{\prime} \cup T R$ & 1003774 & $51.1 / 48.9$ & 0.50 \\
\hline SNP-2 & $A / G$ & 49553 & $\begin{array}{l}\text { gtgaggaaagaacgggtgtt } \\
\text { tctacctggtgccacaacaa }\end{array}$ & ccaagtaccctgcactaggcc & 134 & $5^{\prime} \cup T R$ & 743363 & $46.8 / 53.2$ & 0.50 \\
\hline SNP-4 & $\mathrm{C} / \mathrm{T}$ & 78419 & $\begin{array}{l}\text { aggcagcaagaacctttcaa } \\
\text { ctaaggagctccacgtctgg }\end{array}$ & agtgctccccaccctgagcggaggecccaatggcta & 176 & Tyr/Tyr & Deckert et al ${ }^{c}$ & $57.9 / 42.1$ & 0.49 \\
\hline SNP-5 & $\mathrm{C} / \mathrm{T}$ & 87509 & $\begin{array}{l}\text { cgcctgaataatgtgctggt } \\
\text { gctatgcttcgccctaggat }\end{array}$ & cccactcctctctctgggattc & 121 & $3^{\prime} \cup T R$ & 1041749 & $57.1 / 42.9$ & 0.49 \\
\hline
\end{tabular}

\footnotetext{
aLocation relative to genomic clone AP000355.

bDatabase of SNPs, http://www.ncbi.nlm.nih.gov/SNP/index.html.

cDeckert et al (1998).
} 
primers and deoxynucleotides in the PCR products were then degraded by adding a $5 \mu \mathrm{l}$ solution of $1 \mathrm{U}$ of shrimp alkaline phosphatase (Roche, Indianapolis), $0.5 \mathrm{U}$ of Escherichia coli Exonuclease I (USB, Cleveland), $5 \mathrm{mM} \mathrm{MgCl}_{2}$, and $50 \mathrm{mM}$ Tris- $\mathrm{HCl}(\mathrm{pH} 8.5)$. The mixture was incubated at $37^{\circ} \mathrm{C}$ for $90 \mathrm{~min}$, followed by deactivation for $15 \mathrm{~min}$ at $95^{\circ} \mathrm{C}$. In the final step, a $5 \mu \mathrm{l}$ solution contained $1 \mu \mathrm{M}$ TDI probe (Table 1), $0.4 \mathrm{U}$ of Thermosequenase (USB), $50 \mathrm{mM}$ Tris- $\mathrm{HCl}, 50 \mathrm{mM} \mathrm{KCl}, 5 \mathrm{mM} \mathrm{MgCl}, 5 \mathrm{mM} \mathrm{NaCl}, 8 \%$ glycerol, $125 \mathrm{nM}$ dideoxynucleotides not corresponding to the polymorphic bases, $109.4 \mathrm{nM}$ dideoxynucleotides corresponding to the polymorphic bases, and $15.6 \mathrm{nM} \mathrm{R} 110$ - or TAMRA-labelled dideoxynucleotides corresponding to the polymorphic bases (NEN, Boston) was added to the samples. This mixture was cycled at $95^{\circ} \mathrm{C}$ for $2 \mathrm{~min}$, followed by 35 cycles of $94^{\circ} \mathrm{C}$ for $15 \mathrm{~s}$ and $55^{\circ} \mathrm{C}$ for $30 \mathrm{~s}$. Following template-directed incorporation, $10 \mu \mathrm{l}$ of the sample was transferred to 384-well plates for reading of fluorescence polarization in a TECAN Ultra plate reader (TECAN-US, Research Triangle Park, NC), using dichroic filters provided by the manufacturer. Data output from the reader is in dimensionless units, $\mathrm{mP}$, as previously described (Chen et al, 1999). The data were imported into a software package, EasySNP (TECAN-US, Research Triangle Park, NC), that graphed the data points and four clusters of points were observed for each marker corresponding to homozygotes, heterozygotes, and PCR blank/failed reactions. These clusters were converted to alleles and then imported into LABMAN for storage and further manipulation (Adams, 1994).

\section{Data Analysis}

Linkage analyses. Following a previously proposed strategy (Abreu et al, 1999), we performed parametric linkage analyses assuming a $1 \%$ disease allele frequency and allowing for a reduced penetrance $(50 \%)$ and a phenocopy rate of $1 \%$ for affected individuals (Greenberg et al, 1998; Hodge et al, 1997). Females (males) who were either phenotypically unknown or unaffected had an assumed penetrance model of $66 \%(33 \%)$ with a phenocopy rate of $14 \%(7 \%)$. The genetic parameters for the analyses are based on the results from our previous segregation analyses (Vieland et al, 1993b, 1996). Although these parameters may be crude approximations of the unknown, but true, genetic model for panic disorder, considerable work has shown that the magnitude of the lod score is relatively immune to misspecification of genetic parameters like penetrance or gene frequencies (Hodge et al, 1997). Surprisingly, genetically complex models can be fairly well approximated by singlelocus analyses with reduced penetrance when examining linkage by a single locus at a time (Durner et al, 1999; Vieland et al, 1993a), as performed here. These approximate analyses are less sensitive to the penetrance than to the mode of inheritance at the locus being examined. Thus, linkage can be missed if the 'wrong' (either dominant or recessive) mode of inheritance is chosen for the analysis. Since we did not know which mode of inheritance best fits each locus examined, we performed our analyses twice, once assuming dominant and once assuming a recessive inheritance at that locus. Pedigrees were analyzed for linkage using the FASTLINK package (Cottingham et al,
1993; Schaffer et al, 1994). Calculation of lod scores under genetic heterogeneity was carried out with the HOMOG program (Ott, 1999). In the 'narrow' model, individuals who are diagnosed as having 'definite' or 'probable' PD are coded as affected. The 'intermediate' model adds individuals diagnosed with 'possible' PD, and the 'broad' model adds those with 'any' PD, as previously described (Hamilton et al, 1999). In analyses involving agoraphobia, we used the narrow definition of panic (definite or probable). We then defined the following phenotypes: (1) PD $+\mathrm{AG}$, presence of both PD and AG ( $n=207$ persons coded as affected); (2) PD or AG, presence of PD or AG $(n=358)$; (3) AG alone, excluding persons with comorbid PD $(n=23)$; (4) PD alone, excluding persons with comorbid AG $(n=75)$. An affected sibpair analysis, equivalent to a lod score calculated under simple recessive inheritance, which yields a measure of allele-sharing, was performed using the SIBPAIR program in the ANALYZE package (Terwilliger, 1994).

Association analyses. Triads were analyzed with the transmission disequilibrium test (TDT) statistic (Spielman et al, 1993; Terwilliger, 1995) and the Haplotype-based relative risk (HHRR) method (Falk and Rubinstein, 1987; Terwilliger and Ott, 1992), using the ANALYZE package to test for linkage disequilibrium. Since we used the TDT analysis as another method to test for linkage in the pedigrees, all affected individuals were used in the analysis. Haplotype association analyses were tested using TRANSMIT (Clayton, 1999, 1998), which implements the expectation-maximization algorithm (Excoffier and Slatkin, 1995). TRANSMIT compares the transmission of multilocus haplotypes from parents to affected offspring. Its main advantage is its ability to handle missing parental genotypes or phase-unknown parental genotypes. TRANSMIT reports a global $\chi^{2}$ test of transmission distortion with $h-1$ degrees of freedom, where $h$ is the number of haplotypes for which transmission data are available. Global analysis addresses the null hypothesis that none of the haplotypes are associated with panic disorder. Individual analyses test the null hypothesis that haplotype is not associated with PD. Thus, the individual analyses will have more statistical power than the global hypothesis. Individual analyses would be the preferred test, but we do not know which haplotype to test so we test them all. Since we test them all, this raises the issue of multiple comparisons, so we need to interpret the results carefully and most likely adjust the significance level. Specific haplotype tests of transmission distortion are also reported using a one-degree of freedom $\chi^{2}$ test. We did not construct haplotypes for the pedigrees, as there is no adequate software for inferring appropriate haplotypes for our pedigree data. For example, TRANSMIT only constructs haplotypes for nuclear families, while GENEHUNTER has a peculiar propensity to arbitrarily choose only one of several possible haplotypes (Lindholm et al, 2003; Schaid et al, 2002).

Using the triad data, we tested for linkage disequilibrium and estimated the strength of disequilibrium for all pairwise marker combinations. The likelihood ratio test and parameters estimates were obtained from the GOLD computer program (Abecasis and Cookson, 2000). As a measure of linkage disequilibrium, we report $D^{\prime}$, a normalized linkage disequilibrium parameter (Devlin and Risch, 1995). 


\section{RESULTS}

\section{Linkage Analyses}

A total of 70 multiplex PD pedigrees containing 620 individuals were genotyped with five biallelic SNPs in a $39.5 \mathrm{~kb}$ region encompassing ADORA2A. Three of the SNPs are in the $5^{\prime}$ untranslated region, one is in exon 2 , and one is in the $3^{\prime}$ untranslated region (Table 1). Although most work describes the ADORA2A gene as containing two exons, one reported sequence contains three additional untranslated exons farther upstream (GenBank accession X68486), and SNPs $1-3$ in our paper lie $5^{\prime}$ to the first of these additional exons. Minor allele frequencies in 140 founders averaged 0.37 , with a range of $0.029-0.489$ (Table 1 ). We analyzed each marker for linkage in the following ways: three diagnostic models, two genetic models, and two homogeneity $v s$ heterogeneity models, in addition to affected sibpair analyses. As described below, parametric linkage analyses were also performed by considering agoraphobia in the phenotype. Using the homogeneity model, SNP-4, a silent coding variant in exon 2, showed elevated lod scores in all recessive models (broad (1.66), intermediate (1.87), narrow (2.95) phenotype). No other marker showed a maximal homogeneity lod score $>1.0$. Calculation of lod scores under genetic heterogeneity, a more realistic model for a complex genetic disorder like PD, increased the lod scores slightly for SNP-4 (1.95 for recessive/intermediate to 2.33 and 2.98, respectively, for recessive/broad and recessive/narrow) (Table 2). The estimates of the proportion of pedigrees linked to SNP-4 $(\alpha)$ ranged from 0.37 to 0.64 , while the estimates of recombination, $\theta$, for these lod scores were small $(0-0.06)$. Note that although heterogeneity lod scores are valuable for detecting linkage, estimates of $\alpha$ are extremely unreliable for complex disorders (Vieland and Logue, 2002). No other heterogeneity lod scores were $>1.0$, except for a score of 1.16 for SNP-5 under the recessive/ broad model, with $\theta=0$. It is still notable that many lod scores are positive in the same region. 'Nonparametric' analysis using SIBPAIR resulted in nominal $p$-values for SNPs 1 and 4 of 0.005 and 0.001 , respectively, under the narrow diagnostic category. SNP-4 also showed $p$-values of 0.03 and 0.01 , respectively, under the broad and intermediate categories.

We performed family-based association tests on 83 parent-proband triads and on our pedigree sample. TDT and HHRR results for the triads were not significant for any of the SNPs. In the pedigrees, the HHRR test was nonsignificant, but $p$-values of 0.006 and 0.05 from the TDT test results were observed assuming the broad model at SNPs 3 and 5, respectively.

\section{Haplotype Analysis and Linkage Disequilibrium}

We tested for association between ADORA2A SNPs and PD by constructing multilocus haplotypes using the TRANSMIT program. The global $p$-values, which take all possible haplotype combinations into account, were not significant at the 0.05 significance level. Nor were any global $p$-values significant for two-loci and three-loci haplotypes (results not shown). However, significant individual combinations were. For example, the strongest four-loci single haplotype combination included SNPs 2, 3, 4, and 5 (haplotype G/G/C/ $\mathrm{C}, p=0.006)$. This was the third most common haplotype among the nine observed (data not shown). The strongest individual four-marker haplotypes required both SNPs 4 and 5. Again, when all combinations were taken into consideration, no haplotype was significantly associated with PD. The 2-marker haplotype combinations using SNPs 4 and 5 were not significantly associated with panic, while individual 3-marker haplotypes including SNPs 4, 5 and either 1 or 2 were significantly associated, but not when all haplotypes were taken into consideration.

We also assessed the level of linkage disequilibrium between SNPs in the ADORA2A gene by using the GOLD program (Abecasis and Cookson, 2000). Estimates of $D^{\prime}$ between the SNPs are presented in Figure 1. Of the 10 pairwise estimates, six were highly significant $(p<0.00001)$ and four were not significant. All four of the nonsignificant combinations involved the SNP-3, the marker with a low minor allele frequency in this population. SNP-1 showed significant linkage disequilibrium in this sample with markers as far away as SNP-5, almost $40 \mathrm{~kb}$ distant. Hence, from these observations, most of this gene is in disequilibrium and is inherited as a single segment in our sample.

\section{Panic and Agoraphobia as Phenotypes}

Recently, Gelernter et al (2001) performed a genomic screen in a collection of 20 pedigrees segregating $P D$ and agoraphobia (AG). The authors carried out linkage analyses

Table 2 Heterogeneity LOD Scores of Linkage in Panic Disorder Using ADORA2A SNP Genotypes

\begin{tabular}{|c|c|c|c|c|c|c|}
\hline \multirow[b]{2}{*}{ Marker } & \multicolumn{3}{|c|}{ Dominant } & \multicolumn{3}{|c|}{ Recessive } \\
\hline & Narrow & Intermediate & Broad & Narrow & Intermediate & Broad \\
\hline SNP-2 & $0.54,1.00,0.26$ & $0.53,1.00,0.26$ & $0.25,1.00,0.34$ & $0.63,0.29,0$ & $0.06,0.07,0$ & Neg \\
\hline SNP-3 & Neg & Neg & Neg & $0.23,0.34,0$ & Neg & $0.08,0.27,0$ \\
\hline
\end{tabular}

LOD scores greater than 1.0 are indicated in bold. For each SNP-model combination we present, in order, $\mathrm{Zmax}-\mathrm{H}$, , and . Data are presented for dominant and recessive genetic models, with narrow, intermediate, and broad diagnostic categories. When heterogeneity LOD scores were negative (Neg), no estimate of $\alpha$ was made. Abbreviations: Zmax-H, Maximum LOD score under heterogeneity; $\alpha$, estimated proportion of families linked at Zmax-H; $\theta$, estimated recombination fraction at Zmax. 


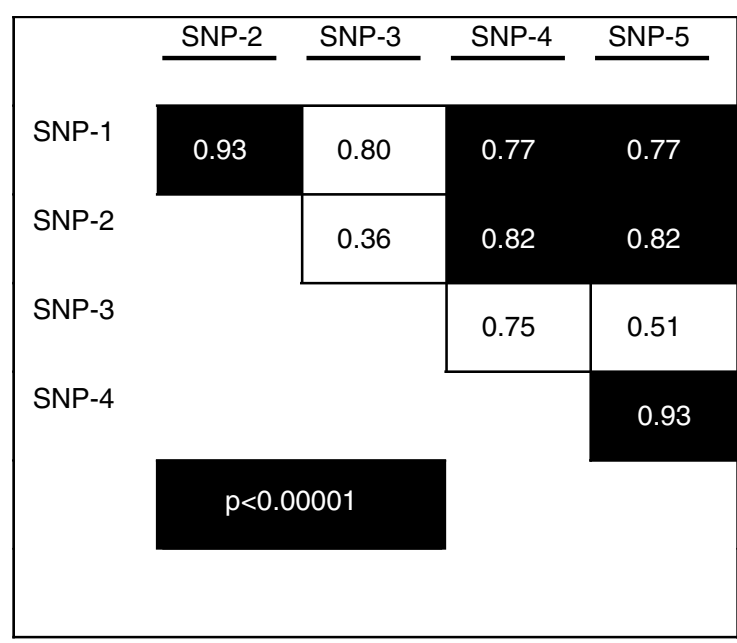

Figure I Pairwise linkage disequilibrium $\left(D^{\prime}\right)$ estimations using GOLD Numbers in boxes represent estimations of $D^{\prime}$. Dark shading represents $p<0.00001$.

using either PD and/or AG as the phenotype. Interestingly, their results suggest some loci common to both diagnoses as well as loci specific to one disorder and not the order. As we have data on AG, we have taken a similar approach. We performed linkage analyses by changing proband affected status depending on $\mathrm{AG}$ status. Interestingly, when we examined these phenotypes for linkage to five ADORA2A SNP loci, we observed several lod scores above 1.0, with one heterogeneity lod score of 3. Remarkably, the addition of the agoraphobia phenotype raised scores in the more $5^{\prime}$ ADORA2A SNPs. For example, for the PD + AG phenotype, heterogeneity lod scores $>1.0$ are seen at three of the five markers $(1.33,1.58$, and 1.12, respectively, for SNPs-1, 2, and 4). The broader PD or AG phenotype showed scores $>1.0$ at four markers, with a heterogeneity lod score of 3.2 at SNP-4 using a recessive genetic model $(\theta=0$, $\alpha=0.63)$. SNPs 1,2 , and 5 showed scores $>1$ using the same model. Analysis of AG alone and PD alone showed little support for linkage, with the exception of a homogeneity lod score of 1.43 at SNP-2 for AG alone. The small number of affecteds for these analyses $(n=23$ for AG alone, $n=75$ for PD alone), as compared to $\mathrm{PD}+\mathrm{AG}(n=207)$ and PD or AG $(n=358)$ may explain these findings.

\section{DISCUSSION}

The work here provides promising evidence for linkage between the ADORA2A locus and PD. Using biallelic SNPs, we observed that the site of a $\mathrm{C} / \mathrm{T}$ transition in exon 2 (SNP4) showed suggestive linkage to PD. This SNP has been previously reported to be associated to PD when 89 unrelated German PD probands were compared to 89 controls (Deckert et al, 1998). However, our own sample showed no significant association. Nevertheless, when our linkage data are viewed together with the association data of Deckert et al, the ADORA2A gene is the first gene for $\mathrm{PD}$ that has been implicated in multiple studies. We observed that under different genetic models, different phenotypic categories were significantly linked to markers. The results likely differ because the analyses use the pedigree data differently. Sibpair analysis and TDT break up the pedigrees, whereas the linkage program analyzes the pedigrees as a whole unit. It is not clear to us why the TDT found SNP-3 to be significant but Sibpair analysis and linkage did not. We suggest that with complex disorders, no single model captures the inherent complexity of the underlying genetic architecture.

We are intrigued that adding the AG diagnosis to $\mathrm{PD}$, essentially broadening the phenotype, increased the lod scores at all of the markers in the gene, and increased the positive score seen at SNP-4. This finding is interesting in light of clinical observations. The adenosine receptor is linked to the pharmacological effects of caffeine (Dunwiddie and Masino, 2001). Caffeine has been reported as anxiogenic in normal subjects and in those with anxiety or depressive disorders (Lee et al, 1988; Nickell and Uhde, 1994). Further, intravenous caffeine increases HPA activity, as shown by increased ACTH and cortisol (Lin et al, 1997). This does not occur during the clinical panic attack but does occur with anticipatory anxiety. This suggests that the effect of caffeine may be more closely related to chronic anxiety and that caffeine is not associated with the panic attack itself. An approach to this issue to compare pure panic patients without $A G$ to patients with both $A G$ and $P D$, since only the latter have clearly increased chronic anxiety. The hypothesis is that the panic/AG group would show linkage to the adenosine receptor, but the pure panic group would not. Our data are supportive of this hypothesis, but are far from conclusive given the small number of individuals in our sample with 'pure' PD. One reasonable way to test this would be to perform a caffeine challenge test and then stratify the sample by genotype. Recently, this was carried out in infrequent caffeine users (Alsene et al, 2003). Volunteers were administered $150 \mathrm{mg}$ of caffeine or placebo and then measured for various mood and anxiety measures. These subjects were also genotyped for several SNPs in ADORA2A. The authors observed a rapid and significant increase in anxiety measures in subjects homozygous for the T allele of an SNP ('1976C/T'), which is in fact the same as SNP-4 in this work. Individuals who were $\mathrm{C} / \mathrm{T}$ were similar to those who were C/C. An alternative interpretation might be that since caffeine sensitivity is seen in a number of anxiety disorders and depression, casting a broader phenotypic net might actually capture an intermediate phenotype that crosses standard diagnostic categories.

This is not the first study of the genetics of PD in which 'broadening' the phenotype from DSM PD increases the evidence for the presence of a gene for the disorder. As mentioned above, a recent genome scan reported data using $\mathrm{PD}$ or AG as phenotypes (Gelernter et al, 2001). In this study, positive lod scores were obtained at a number of different loci when PD and AG were analyzed separately. When a phenotype combining AG and/or PD (like our 'PD or AG') was used, several lod scores $>1.0$ were observed, although not on chromosome 22, the location of ADORA2A. Another group, looking at genomic regions that are syntenic with regions of the mouse genome linked to anxiety quantitative trait loci, found evidence for linkage between chromosome $12 \mathrm{q}$ and the $\mathrm{PD}$ and/or AG phenotype (Smoller et al, 2001). By broadening the phenotype even more, including comorbid anxiety disorder, childhood anxiety disorders, or continuous anxiety disorder diagnosis 
since age 13, the authors observed evidence for linkage to chromosome 10q. Finally, a Spanish group detected the presence of a large interstitial duplication of chromosome 15q24-26 in pedigrees segregating $P D$ and joint laxity (Gratacos et al, 2001), a finding that was not replicated in another sample (Tabiner et al, 2003). Oddly, evidence for linkage between this duplication and panic occurred when the phenotype was maximally broadened to comprise PD, $A G$, joint laxity, and social phobia (lod $\sim 5$ ). The data from these studies and from the findings presented here suggest that the phenotypes of interest may cross the diagnostic boundaries of both psychiatry and medicine, reflecting genetic vulnerability to a spectrum of illness.

It is also possible that we have not actually 'broadened' the phenotype presented here. For instance, given the association between panic severity, frequency, and duration with AG (Katschnig and Amering, 1998), it could be suggested that adding the probands with AG to the phenotype increases sensitivity by including a larger number of more severely affected subjects. This may represent adding the most significantly genetically loaded individuals with less admixing of unrelated phenotypes. In this view, our findings may suggest that ADORA2A is associated with a particular type of $\mathrm{PD}$ with more severe expression or, at least that is more likely to result in AG.

We have previously published results of similar magnitude from another locus on chromosome 22. With the same pedigree set, we found linkage and association between PD and markers in or near the catechol- $O$-methyltransferase gene on 22q11.2, some 4.6 million bases centromeric to ADORA2A (Hamilton et al, 2002). It is unlikely that the results presented here are due to variation at COMT or another gene in between COMT and ADORA2, but we cannot rule out that possibility, since our strongest findings are with linkage tests. Likewise, there are two genes within $100 \mathrm{~kb}$ centromeric or telomeric to ADORA2A, the predicted gene KIAA0376 and beta-ureidopropionase (UPB1), respectively. Although these genes are not obvious candidates for $\mathrm{PD}$, linkage disequilibrium between SNPs at the ADORA2A locus and one of these other genes may explain our findings.

As for the haplotype analyses using TRANSMIT, a few specific haplotypes were preferentially transmitted with PD at a suggestive level of significance $(p<0.05)$. Specifically, there is the evidence of 3-marker combinations of SNPs 1, 4, 5 or $2,4,5$. It therefore appears that SNPs 4 and 5 are required, with either SNP-1 or SNP-2 needed to add information. But markers 4 and 5 are not informative enough by themselves to yield statistically significant results in this dataset. Likewise, the low-frequency SNP-3 appears to add information to the $2,4,5$ haplotype. We note, however, that the global $p$-values of these analyses were not significant and that we tested many different haplotypes. These $p$-values, therefore, must be interpreted with caution.

Although SNP-4 does not alter the amino-acid sequence of ADORA2A, the substitution of $\mathrm{T}$ for $\mathrm{C}$ results in the TAT codon, which appears much less often than the TAC codon (Nakamura et al, 2000) and could possibly alter mRNA processing or the translational efficiency of the gene. There is both theoretical and biological evidence for the role of socalled 'silent' variants in functional variation. For instance, analysis of two tyrosine codons in a consensus sequence among 71 olfactory receptors revealed that the codon preference for TAT at one residue was $>90 \%\left(p<10^{-19}\right)$, while a nearby second tyrosine residue shows $\sim 60 \% \mathrm{TAT}$, much closer to the expected distribution (Conticello et al, 2000). Recent work in Drosophila highlights biological evidence for the functional effects of codon bias. Carlini and Stephan (2003) introduced the less preferred codon for leucine in a number of leucine residues in the Drosophila alcohol dehydrogenase gene. The introduction of the unpreferred codon led to a significant decrease in enzyme activity. The authors argued that the effect was likely not due to mRNA structural changes, and was likely due to translational effects.

When comparing our current data with that of Deckert et al (1998), we note a difference between results. In the case-control study of Deckert et al, positive association was noted with the $\mathrm{T}$ allele of the $1083 \mathrm{C} / \mathrm{T}$ polymorphism (SNP4 in this report). In the three individual haplotypes showing significant association in our study (SNPs 2, 3, 4, 5-GGCC, SNPs 1, 3, 4, 5-GGCC, SNPs 1, 2, 4, 5-GGCC), the identity for SNP-4 was always C. This suggests that SNP-4 itself is not the disease allele, but may be in proximity to, or in linkage disequilibrium with, a DNA variation that contributes to PD susceptibility. Which allele cosegregates with disease in our pedigrees? Linkage analysis focuses on loci, not on particular alleles. Our linkage analysis yielded significant findings at this locus, but our association analysis did not. This explains why some of the pedigrees exhibited cosegregation of $\mathrm{PD}$ with the $\mathrm{C}$ allele, others with the $\mathrm{T}$ allele. In a Japanese population of 87 probands and 99 controls, no association was seen between this variant and PD (Yamada et al, 2001), a finding plausibly due to specific population histories that may be reflected in the extent of linkage disequilibrium in the region. Such an explanation invoking linkage disequilibrium must be viewed as a provisional limitation in interpreting the significance of our findings, although this study still represents a rare replication of a candidate gene study for a candidate gene of great functional significance.

\section{ACKNOWLEDGEMENTS}

We acknowledge the invaluable contribution made by the families who participated in this study. We also acknowledge TECAN-US for the use of an ULTRA plate reader. This study was supported by grants: NIMH; MH28274 (MMW), MH37592 (AJF), MH30906 (DFK), and MH48858 (SEH), and an NARSAD Young Investigator Award (SPH). The authors report no involvement, financial or otherwise, that might potentially bias this work. The authors declare no involvement, financial or otherwise, that might potentially bias the work reported here.

\section{REFERENCES}

Abecasis GR, Cookson WO (2000). GOLD - graphical overview of linkage disequilibrium. Bioinformatics 16: 182-183.

Abreu PC, Greenberg DA, Hodge SE (1999). Direct power comparisons between simple LOD scores and NPL scores for linkage analysis in complex diseases. Am J Hum Genet 65: 847-857. 
Adams P (1994). LABMAN and LINKMAN: a data management system specifically designed for genome searches of complex diseases. Genet Epidemiol 11: 87-98.

Alsene K, Deckert J, Sand P, De Wit H (2003). Association between $\mathrm{A}(2 \mathrm{a})$ receptor gene polymorphisms and caffeine-induced anxiety. Neuropsychopharmacology, (advance online publication, 25 June 2003; doi:10.1038/sj.npp.1300232) 28: 1694-1702.

Carlini DB, Stephan W (2003). In vivo introduction of unpreferred synonymous codons into the Drosophila Adh gene results in reduced levels of ADH protein. Genetics 163: 239-243.

Charney DS, Heninger GR, Jatlow PI (1985). Increased anxiogenic effects of caffeine in panic disorders. Arch Gen Psychiatry 42: 233-243.

Chen X, Levine L, Kwok PY (1999). Fluorescence polarization in homogeneous nucleic acid analysis. Genome Res 9: 492-498.

Clayton D (1999). A generalization of the transmission/disequilibrium test for uncertain-haplotype transmission. Am J Hum Genet 65: 1170-1177.

Clayton DG (1998). TRANSMIT [v2.3].

Conticello SG, Pilpel Y, Glusman G, Fainzilber M (2000). Positionspecific codon conservation in hypervariable gene families. Trends Genet 16: 57-59.

Cottingham Jr RW, Idury RM, Schaffer AA (1993). Faster sequential genetic linkage computations. Am J Hum Genet 53: 252-263.

Deckert J, Nothen MM, Franke P, Delmo C, Fritze J, Knapp M et al (1998). Systematic mutation screening and association study of the A1 and A2a adenosine receptor genes in panic disorder suggest a contribution of the A2a gene to the development of disease. Mol Psychiatry 3: 81-85.

Devlin B, Risch N (1995). A comparison of linkage disequilibrium measures for fine-scale mapping. Genomics 29: 311-322.

Dunwiddie TV, Masino SA (2001). The role and regulation of adenosine in the central nervous system. Annu Rev Neurosci 24: 31-55.

Durner M, Vieland VJ, Greenberg DA (1999). Further evidence for the increased power of LOD scores compared with nonparametric methods. Am J Hum Genet 64: 281-289.

Excoffier L, Slatkin M (1995). Maximum-likelihood estimation of molecular haplotype frequencies in a diploid population. $\mathrm{Mol}$ Biol Evol 12: 921-927.

Falk CT, Rubinstein P (1987). Haplotype relative risks: an easy reliable way to construct a proper control sample for risk calculations. Ann Hum Genet 51: 227-233.

Fyer AJ, Weissman MM (1999). Genetic linkage study of panic: clinical methodology and description of pedigrees. Am J Med Genet (Neuropsychiatr Genet) 88: 173-181.

Gelernter J, Bonvicini K, Page G, Woods SW, Goddard AW, Kruger $S$ et al (2001). Linkage genome scan for loci predisposing to panic disorder or agoraphobia. Am J Med Genet 105: 548-557.

Gratacos M, Nadal M, Martin-Santos R, Pujana MA, Gago J, Peral B et al (2001). A polymorphic genomic duplication on human chromosome 15 is a susceptibility factor for panic and phobic disorders. Cell 106: 367-379.

Greenberg DA, Abreu P, Hodge SE (1998). The power to detect linkage in complex disease by means of simple LOD-score analyses. Am J Hum Genet 63: 870-879.

Guieu R, Dussol B, Halimi G, Bechis G, Sampieri F, Berland Y et al (1998). Adenosine and the nervous system: pharmacological data and therapeutic perspectives. Gen Pharmacol 31: 553-561.

Hamilton SP, Heiman GA, Haghighi F, Mick S, Klein DF, Hodge SE et al (1999). Lack of genetic linkage or association between a functional serotonin transporter polymorphism and panic disorder. Psychiatr Genet 9: 1-6.

Hamilton SP, Slager SL, Heiman GA, Deng Z, Haghighi F, Klein DF et al (2002). Evidence for a susceptibility locus for panic disorder near the catechol-O-methyltransferase gene on chromosome 22 . Biolog Psychiatry 51: 591-601.
Hamilton SP, Slager SL, Helleby L, Heiman GA, Klein DF, Hodge SE et al (2001). No association or linkage between polymorphisms in the genes encoding cholecystokinin and cholecystokinin B receptor and panic disorder. Mol Psychiatry 6: 59-65.

Hettema JM, Neale MC, Kendler KS (2001). A review and metaanalysis of the genetic epidemiology of anxiety disorders. Am J Psychiatry 158: 1568-1578.

Hodge SE, Abreu PC, Greenberg DA (1997). Magnitude of type I error when single-locus linkage analysis is maximized over models: a simulation study. Am J Hum Genet 60: 217-227.

Katschnig H, Amering M (1998). The long-term course of panic disorder and its predictors. J Clin Psychopharmacol 18: 6S-11S.

Knowles JA, Fyer AJ, Vieland VJ, Weissman MM, Hodge SE, Heiman GA et al (1998). Results of a genome-wide genetic screen for panic disorder. Am J Med Genet (Neuropsychiatr Genet) 81: 139-147.

Knowles JA, Weissman MM (1995). Panic disorder and agoraphobia. In Oldham JM, Riba MB (eds). Review of Psychiatry, Volume 14. American Psychiatric Press: Washington, DC. Vol. 14, pp 383-404.

Ledent C, Vaugeois JM, Schiffmann SN, Pedrazzini T, El Yacoubi M, Vanderhaeghen JJ et al (1997). Aggressiveness, hypoalgesia and high blood pressure in mice lacking the adenosine A2a receptor. Nature 388: 674-678.

Lee MA, Flegel P, Greden JF, Cameron OG (1988). Anxiogenic effects of caffeine on panic and depressed patients. Am J Psychiatry 145: 632-635.

Lin AS, Uhde TW, Slate SO, McCann UD (1997). Effects of intravenous caffeine administered to healthy males during sleep. Depress Anxiety 5: 21-28.

Lindholm E, Zhang J, Hodge SE, Greenberg DA (2003). The accuracy of haplotype inference in nuclear families: error rates for SNPs and microsatellites. (manuscript submitted).

Mannuzza S, Fyer AJ, Endicott J, Klein DF (1985). Family Informant Schedule and Criteria. Anxiety Disorders Clinic: New York State Psychiatric Institute.

Mannuzza S, Fyer AJ, Klein DF, Endicott J (1986). Schedule for affective disorders and schizophrenia - lifetime version modified for the study of anxiety disorders (SADS-LA): rationale and conceptual development. J Pschiat Res 20: 317-325.

Moreau JL, Huber G (1999). Central adenosine A(2A) receptors: an overview. Brain Res Brain Res Rev 31: 65-82.

Nakamura Y, Gojobori T, Ikemura T (2000). Codon usage tabulated from international DNA sequence databases: status for the year 2000. Nucleic Acids Res 28: 292.

Nickell PV, Uhde TW (1994). Dose-response effects of intravenous caffeine in normal volunteers. Anxiety 1: 161-168.

Ott J (1999). Analysis of Human Genetic Linkage. Johns Hopkins University Press: Baltimore.

Rozen S, Skaletsky HJ (1998). Primer3.

Schaffer AA, Gupta SK, Shriram K, Cottingham Jr RW (1994). Avoiding recomputation in linkage analysis. Hum Hered 44: 225-237.

Schaid DJ, McDonnell SK, Wang L, Cunningham JM, Thibodeau SN (2002). Caution on pedigree haplotype inference with software that assumes linkage equilibrium. Am J Hum Genet 71: 992-995.

Sebastiao AM, Ribeiro JA (2000). Fine-tuning neuromodulation by adenosine. Trends Pharmacol Sci 21: 341-346.

Smoller JW, Acierno Jr JS, Rosenbaum JF, Biederman J, Pollack $\mathrm{MH}$, Meminger $\mathrm{S}$ et al (2001). Targeted genome screen of panic disorder and anxiety disorder proneness using homology to murine QTL regions. Am J Med Genet 105: 195-206.

Spielman RS, McGinnis RE, Ewens WJ (1993). Transmission test for linkage disequilibrium: the insulin gene region and insulindependent diabetes mellitus (IDDM). Am J Hum Genet 52: 506-516. 
Tabiner M, Youings S, Dennis N, Baldwin D, Buis C, Mayers A et al (2003). Failure to find DUP25 in patients with anxiety disorders, in control individuals, or in previously reported positive control cell lines. Am J Hum Genet 72.

Terwilliger JD (1994). The available possibilities to analyze data of polygenic disease statistically. Abstract to IVth Workshop of the Nordic Genome Initiative, Helsinki.

Terwilliger JD (1995). A powerful likelihood method for the analysis of linkage disequilibrium between trait loci and one or more polymorphic marker loci. Am J Hum Genet 56: 777-787.

Terwilliger JD, Ott J (1992). A haplotype-based 'haplotype relative risk' approach to detecting allelic associations. Hum Hered 42: 337-346.

Vieland VJ, Goodman DW, Chapman T, Fyer AJ (1996). New segregation analysis of panic disorder. Am J Med Genet 67: 147-153.
Vieland VJ, Greenberg DA, Hodge SE (1993a). Adequacy of singlelocus approximations for linkage analyses of oligogenic traits: extension to multigenerational pedigree structures. Hum Hered 43: $329-336$.

Vieland VJ, Hodge SE, Lish JD, Adams P, Weissman MM (1993b). Segregation analysis of panic disorder. Psychiatr Genet 3: 63-71.

Vieland VJ, Logue M (2002). HLODs, trait models, and ascertainment: implications of admixture for parameter estimation and linkage detection. Hum Hered 53: 23-35.

Yamada K, Hattori E, Shimizu M, Sugaya A, Shibuya H, Yoshikawa $\mathrm{T}$ (2001). Association studies of the cholecystokinin B receptor and $\mathrm{A} 2 \mathrm{a}$ adenosine receptor genes in panic disorder. $J$ Neural Transm 108: 837-848. 